\title{
Missense mutations in the TP53 DNA-binding domain predict outcomes in patients with advanced oral cavity squamous cell carcinoma
}

\author{
Nina Lapke ${ }^{1, *}$, Yen-Jung Lu' ${ }^{1, *}$, Chun-Ta Liao ${ }^{2, *}$, Li-Yu Lee ${ }^{3}$, Chien-Yu Lin ${ }^{4}$, Hung- \\ Ming Wang ${ }^{5}$, Shu-Hang Ng $^{6}$, Shu-Jen Chen ${ }^{1}$, Tzu-Chen Yen ${ }^{7}$ \\ ${ }^{1}$ ACT Genomics, Taipei, Taiwan, ROC \\ ${ }^{2}$ Department of Otorhinolaryngology, Head and Neck Surgery, Chang Gung Memorial Hospital and Chang Gung University, \\ Taoyuan, Taiwan, ROC \\ ${ }^{3}$ Department of Pathology, Chang Gung Memorial Hospital and Chang Gung University, Taoyuan, Taiwan, ROC \\ ${ }^{4}$ Department of Radiation Oncology, Chang Gung Memorial Hospital and Chang Gung University, Taoyuan, Taiwan, ROC \\ ${ }^{5}$ Department of Medical Oncology, Chang Gung Memorial Hospital and Chang Gung University, Taoyuan, Taiwan, ROC \\ ${ }^{6}$ Department of Diagnostic Radiology, Chang Gung Memorial Hospital and Chang Gung University, Taoyuan, Taiwan, RoC \\ ${ }^{7}$ Department of Nuclear Medicine and Molecular Imaging Center, Chang Gung Memorial Hospital and Chang Gung University, \\ Taoyuan, Taiwan, ROC \\ *These authors have contributed equally to this work \\ Correspondence to: Shu-Jen Chen, email: sjchen@actgenomics.com \\ Tzu-Chen Yen, email: yen $1110 @ a d m . c g m h . o r g . t w$ \\ Keywords: oral cavity squamous cell carcinoma, missense mutations, TP53 DNA-binding domain, biomarker, outcome \\ Received: March 21, $2016 \quad$ Accepted: May 13, $2016 \quad$ Published: June 8, 2016
}

\section{ABSTRACT}

TP53 mutations have been linked to reduced survival in patients with oral cavity squamous cell carcinoma (OSCC). However, the impact of different types of TP53 mutations remains unclear. Here, we demonstrate that the carriage of missense mutations in the TP53 DNA binding domain (DBD missense mutations) is associated with decreased disease-specific survival (DSS) compared with wild-type TP53 $(P=0.002)$ in a cohort of 345 OSCC patients. In contrast, DSS of patients bearing all of the remaining TP53 mutations did not differ from that observed in wild-type TP53 patients $(P=0.955)$. Our classification method for TP53 mutations was superior to previously reported approaches (disruptive, truncating, Evolutionary Action score, mutations in L2/L3/LSH) for distinguishing between low- and high-risk patients. When analyzed in combination with traditional clinicopathological factors, TP53 DBD missense mutations were an independent prognostic factor for shorter DSS $(P=0.014)$ alongside with advanced $A J C C$ T- and $N$-classifications and the presence of extracapsular spread. A scoring system that included the four independent prognostic factors allowed a reliable patient stratification into distinct risk groups (high-risk patients, 16.2\%). Our results demonstrate the usefulness of TP53 DBD missense mutations combined with clinicopathological factors for improving the prognostic stratification of OSCC patients.

\section{INTRODUCTION}

Approximately 300,000 new cases of oral cavity cancer are diagnosed each year, with this malignancy being responsible for 150,000 deaths annually (GLOBOCAN 2012, http://globocan.iarc.fr). The main risk factors for oral cavity cancer include cigarette smoking, alcohol drinking [1], and betel nut chewing [2], the latter being highly prevalent in Southeast Asia. Oral cavity squamous cell carcinoma (OSCC) accounts for more than $90 \%$ of all oral cavity malignancies. Unfortunately, 5-year survival rates of patients with advanced OSCC remain poor [3]. TP53 is the most commonly mutated gene in OSCC $(60-80 \%$ of cases) $[4,5]$. Although OSCC patients 
carrying TP53 mutations have reduced survival compared to those with a wild-type status $[6,7]$, the prognostic impact of different types of TP53 mutations remains poorly understood. A commonly used classification is based on a large study conducted by Poeta et al. [8] that enrolled 560 patients with squamous cell carcinoma of the head and neck (HNSCC). In this study, all of the mutations that introduced a stop codon or non-conservative mutations in specific DNA binding domains (DBDs) were defined as disruptive. Disruptive TP53 mutations are associated with a significantly decreased survival $[8,9]$. However, disruptive mutations include two biologically different subtypes, namely 1) truncating mutations associated with a loss of tumor suppressive activity, and 2) DBD missense mutations. Although truncating mutations have been associated with an unfavorable prognosis [9], further confirmation of these findings is necessary. DBD missense mutations can result in a gain-of-function, ultimately leading to cell invasion, migration, proliferation, and drug resistance [10]. Possible mechanisms leading to a gainof-function include changes in DNA binding properties [11] and/or altered protein-protein interactions [12]. Notably, a study in breast cancer patients demonstrated that only TP53 DBD missense mutations (and not other mutations) have an adverse prognostic impact [13]. It has been recently suggested that TP53 missense mutations occurring in evolutionary conserved residues are likely to confer a gain-of-function, ultimately predicting poor treatment response and a shorter survival in HNSCC patients $[14,15]$. Other studies focusing on mutations occurring in the DBD or DBD-defined regions (e.g., L2, L3 and LSH) [7, 16] have reported their adverse prognostic significance, although conflicting results exist $[7,9,16,17]$. Such discrepancies can be ascribed to small sample sizes or sequencing areas limited to exons 5-8.

Starting from these premises, we designed the current study to shed more light on the prognostic impact of different TP53 mutation types in a large cohort of 345 patients with advanced (AJCC stage III/IV) OSCC (Figure 1). Ultra-deep targeted sequencing (average sequencing depth $>2000 \times$ ) of formalin-fixed paraffinembedded (FFPE) tumor samples was performed for exons $2,4-8$, and 10 . These regions covered all of the relevant hotspots for head and neck cancer identified in the TCGA HNSCC cohort. Working from the assumption that most DBD missense mutations can lead to a gain-offunction $[10,18]$, we categorized TP53 mutations into two distinct categories, i.e., DBD missense mutations versus

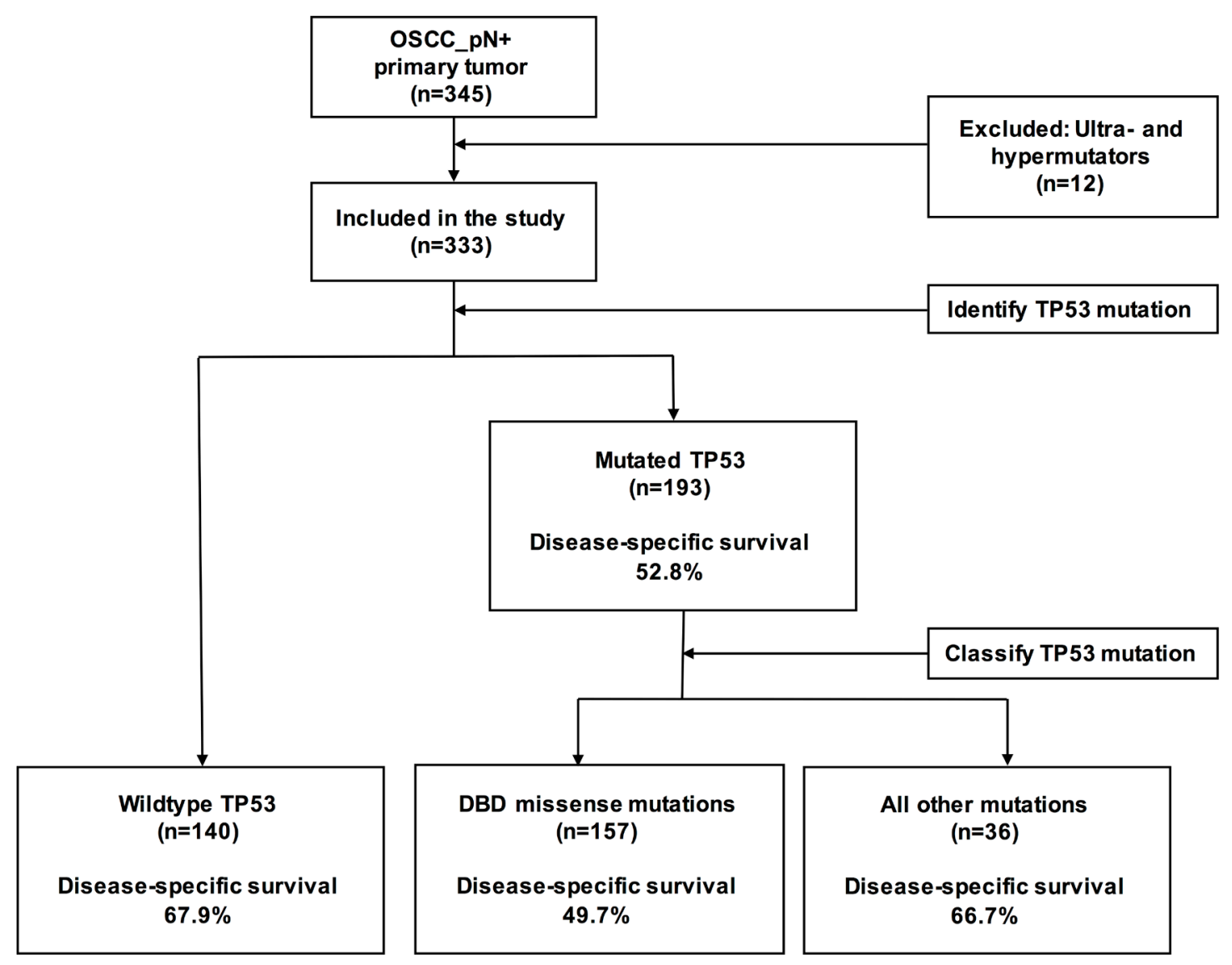

Figure 1: Flow of OSCC patients through the study and TP53 mutation analysis. 
all other mutations. We then compared the predictive value of TP53 DBD missense mutations versus other types of TP53 mutations in terms of disease-specific survival (DSS). Furthermore, we combined TP53 DBD missense mutations with traditional risk factors with the aim of identifying high-risk patients.

\section{RESULTS}

\section{Patient characteristics}

The general characteristics of the study patients are listed in Table 1. In line with previous methodology [19], ultra- and hypermutators $(n=12)$ were excluded. A total of 333 patients were eligible for the study. There was a clear preponderance of male subjects $(94.0 \%, \mathrm{n}=313)$. The median age was 48 years (range: $27-89$ years) and the median follow-up time after surgery was 50 months. The distribution of known risk factors for OSCC was as follows: pre-operative smoking $(90.4 \%, n=301)$, pre-operative betel nut chewing $(81.4 \%, \mathrm{n}=271)$, preoperative alcohol drinking $(71.2 \%, \mathrm{n}=237)$, and HPV16/18 infections (12.6\%, $n=42$; subjects with known HPV16/18 status: $\mathrm{n}=317)$. The tumor sites were as follows: buccal $(38.1 \%, \mathrm{n}=127)$, retromolar trigone $(4.5 \%, \mathrm{n}=15)$, lip $(0.6 \%, \mathrm{n}=2)$, tongue $(37.5 \%, \mathrm{n}=125)$, alveolar ridge $(12.9 \%, \mathrm{n}=43)$, hard palate $(1.8 \%, \mathrm{n}=6)$, and floor of mouth $(4.5 \%, n=15)$. Extracapsular spread (ECS) was observed in $58.0 \%(\mathrm{n}=193)$ of the study patients.

\section{TP53 mutations}

TP53 mutations were observed in 58.0\% $(\mathrm{n}=193)$ of the study patients, with 228 mutations being identified (because two patients harbored three mutations and 31 patients two mutations). The following types of TP53 mutations were observed: missense $(78.1 \%, \mathrm{n}=178)$, stopgain $(15.4 \%, \mathrm{n}=35)$, splice site $(3.5 \%, \mathrm{n}=8)$, frameshift deletions $(1.8 \%, n=4)$, and inframe deletions $(1.3 \%, n=3)$. A total of 68 different mutated amino acids were identified. A list of all of the observed mutations is provided in the Supplementary Tables 1 and 2. The general characteristics of the study participants are reported in Supplementary Table 3. In total, $81.3 \%(n=157)$ of patients with mutated TP53 harbored a TP53 DBD missense mutation. Most $(98.3 \%, \mathrm{n}=175)$ missense mutations occurred in the DBD, with high frequencies being observed for the hotspots R273 (13.5\%, n=24), R248 (11.2\%, n=20), and R175 $(9.6 \%, \mathrm{n}=17)$.

\section{Survival in OSCC patients in relation to the presence of TP53 mutations}

We then compared the characteristics of patients harboring a TP53 mutation (regardless of the mutation type) with those having wild-type TP53 (Table 1). TP53 mutations were associated with alcohol drinking, a margin status of less than $5 \mathrm{~mm}$ and a higher rate of distant metastases $(\mathrm{P}=0.038, \mathrm{P}=0.017$, and $\mathrm{P}=0.002$, respectively). DFS was not significantly lower in patients with mutated TP53 compared with wild-type TP53 (HR, 1.28; 95\% CI, 0.94-1.75; $\mathrm{P}=0.124$; Figure $2 \mathrm{~A})$. However, DSS and OS were found to differ significantly between the two groups (DSS: HR, 1.62; 95\% CI, 1.15-2.25; $\mathrm{P}=0.007$, OS: HR, 1.59 ; 95\% CI, 1.20-2.09; $\mathrm{P}=0.002$; Figure $2 \mathrm{~B}$ and $2 \mathrm{C}$ ).

\section{TP53 DBD missense mutations allow an optimal stratification of TP53-mutant patients}

Based on the assumption that TP53 DBD missense mutations would lead to a gain-of-function, we hypothesized that this mutation subtype may lead to dismal outcomes. We therefore divided patients with TP53 mutations into two subgroups (i.e., TP53 DBD missense mutations versus all other mutations, Figure 3A). TP53 DBD missense mutations were associated with a decreased DSS compared to wild-type TP53 (HR, 1.78; 95\% CI, 1.23-2.57; $\mathrm{P}=0.002$; Table 2). However, DSS of patients with all other mutations was comparable to patients with wild-type TP53 (HR, 1.02; 95\% CI, 0.54-1.93; $\mathrm{P}=0.955$ ).

We then compared the performance of our classification with that of other previously reported methods. Disruptive and truncating mutations were not associated with a significantly decreased DSS compared with patients with wild-type TP53 (disruptive: HR, 1.38; 95\% CI, 0.88-2.15; $\mathrm{P}=0.158$, truncating: HR, 1.45; 95\% CI, 0.85-2.48; $\mathrm{P}=0.177$ ). In contrast, Evolutionary Action score (EAp53) high-risk mutations and mutations in the L2, L3 or LSH regions were good predictors of a shorter DSS compared with wild-type TP53 (EAp53: HR, 1.99; 95\% CI, 1.35-2.93; P=0.001, L2, L3, LSH: 1.78 ; $95 \%$ CI, 1.21-2.59; $\mathrm{P}=0.003)$. However, the DSS of patients harboring TP53 mutations not included in these classifications was also reduced compared with wild-type TP53, albeit not significantly so (low-risk EAp53: HR, $1.49 ; 95 \%$ CI, 0.90-2.49; $\mathrm{P}=0.124$, mutations outside of the L2, L3 or LSH regions: HR, 1.31; 95\% CI, 0.80-2.16; $\mathrm{P}=0.276$ ). Consequently, only DBD missense mutations allowed a clear identification of patients with a poor prognosis compared with wild-type TP53.

\section{TP53 DBD missense mutations are associated with decreased DFS, DSS, and OS}

Because TP53 DBD missense mutations allowed the best discrimination between low- and high-risk TP53 mutation subgroups, we performed a detailed analysis of their association with other survival endpoints (Figure 3B3G). Similar to DSS, a carriage of TP53 DBD missense mutations was associated with a significantly decreased DFS compared with wild-type TP53 (HR, 1.38; 95\% CI, 1.01-1.92; $\mathrm{P}=0.049$; Figure $3 \mathrm{~B})$. In keeping with the 
Table 1: General characteristics of the study patients $(n=333)$ according the TP53 mutation status

\begin{tabular}{|c|c|c|c|c|c|c|c|}
\hline \multirow[t]{2}{*}{ Characteristics } & \multicolumn{2}{|c|}{ Entire cohort } & \multicolumn{2}{|c|}{ TP53 Wt } & \multicolumn{2}{|c|}{ TP53 mutations } & \multirow[t]{2}{*}{ P value } \\
\hline & $\mathbf{N}$ & $(\%)$ & $\mathbf{N}$ & $(\%)$ & $\mathbf{N}$ & $(\%)$ & \\
\hline Entire study cohort & 333 & $(100.0 \%)$ & 140 & $(42.0 \%)$ & 193 & $(58.0 \%)$ & \\
\hline Sex & & & & & & & 0.249 \\
\hline Male & 313 & $(94.0 \%)$ & 129 & $(92.1 \%)$ & 184 & $(95.3 \%)$ & \\
\hline Female & 20 & $(6.0 \%)$ & 11 & $(7.9 \%)$ & 9 & $(4.7 \%)$ & \\
\hline Age, years & & & & & & & \\
\hline Median & 48 & & 47 & & 49 & & \\
\hline Range & 27 & -89 & 29 & -89 & 27 & -83 & \\
\hline Mean \pm SD & 49.7 & \pm 11.0 & 49.5 & \pm 11.7 & 49.9 & \pm 10.6 & \\
\hline Age (categorical) & & & & & & & 0.167 \\
\hline$<65$ years & 295 & $(88.6 \%)$ & 120 & $(85.7 \%)$ & 175 & $(90.7 \%)$ & \\
\hline$\geq 65$ years & 38 & $(11.4 \%)$ & 20 & $(14.3 \%)$ & 18 & $(9.3 \%)$ & \\
\hline Cigarette smoking & & & & & & & 0.577 \\
\hline No & 32 & $(9.6 \%)$ & 15 & $(10.7 \%)$ & 17 & $(8.8 \%)$ & \\
\hline Yes & 301 & $(90.4 \%)$ & 125 & $(89.3 \%)$ & 176 & $(91.2 \%)$ & \\
\hline Betel chewing & & & & & & & 0.318 \\
\hline No & 62 & $(18.6 \%)$ & 30 & $(21.4 \%)$ & 32 & $(16.6 \%)$ & \\
\hline Yes & 271 & $(81.4 \%)$ & 110 & $(78.6 \%)$ & 161 & $(83.4 \%)$ & \\
\hline Alcohol drinking & & & & & & & 0.038 \\
\hline No & 96 & $(28.8 \%)$ & 49 & $(35.0 \%)$ & 47 & $(24.4 \%)$ & \\
\hline Yes & 237 & $(71.2 \%)$ & 91 & $(65.0 \%)$ & 146 & $(75.6 \%)$ & \\
\hline HPV16/18 positive & & & & & & & 0.403 \\
\hline No & 275 & $(82.6 \%)$ & 111 & $(79.3 \%)$ & 164 & $(85.0 \%)$ & \\
\hline Yes & 42 & $(12.6 \%)$ & 20 & $(14.3 \%)$ & 22 & $(11.4 \%)$ & \\
\hline Unknown & 16 & $(4.8 \%)$ & 9 & $(6.4 \%)$ & 7 & $(3.6 \%)$ & \\
\hline Tumor site & & & & & & & 0.290 \\
\hline Buccal & 127 & $(38.1 \%)$ & 61 & $(43.6 \%)$ & 66 & $(34.2 \%)$ & \\
\hline Retromolar Trigone & 15 & $(4.5 \%)$ & 3 & $(2.1 \%)$ & 12 & $(6.2 \%)$ & \\
\hline Lip & 2 & $(0.6 \%)$ & 1 & $(0.7 \%)$ & 1 & $(0.5 \%)$ & \\
\hline Tongue & 125 & $(37.5 \%)$ & 46 & $(32.9 \%)$ & 79 & $(40.9 \%)$ & \\
\hline Alveolar ridge & 43 & $(12.9 \%)$ & 21 & $(15.0 \%)$ & 22 & $(11.4 \%)$ & \\
\hline Hard palate & 6 & $(1.8 \%)$ & 2 & $(1.4 \%)$ & 4 & $(2.1 \%)$ & \\
\hline Mouth floor & 15 & $(4.5 \%)$ & 6 & $(4.3 \%)$ & 9 & $(4.7 \%)$ & \\
\hline AJCC T-classification & & & & & & & 0.147 \\
\hline pT1-2 & 148 & $(44.4 \%)$ & 69 & $(49.3 \%)$ & 79 & $(40.9 \%)$ & \\
\hline pT3-4 & 185 & $(55.6 \%)$ & 71 & $(50.7 \%)$ & 114 & $(59.1 \%)$ & \\
\hline
\end{tabular}




\begin{tabular}{|c|c|c|c|c|c|c|c|}
\hline \multirow[t]{2}{*}{ Characteristics } & \multicolumn{2}{|c|}{ Entire cohort } & \multicolumn{2}{|c|}{ TP53 Wt } & \multicolumn{2}{|c|}{ TP53 mutations } & \multirow[t]{2}{*}{ P value } \\
\hline & $\mathbf{N}$ & $(\%)$ & $\mathbf{N}$ & $(\%)$ & $\mathbf{N}$ & $(\%)$ & \\
\hline $\begin{array}{l}\text { AJCC } \\
\text { N-classification }\end{array}$ & & & & & & & 0.297 \\
\hline pN1 & 119 & $(35.7 \%)$ & 55 & $(39.3 \%)$ & 64 & $(33.2 \%)$ & \\
\hline $\mathrm{pN} 2$ & 214 & $(64.3 \%)$ & 85 & $(60.7 \%)$ & 129 & $(66.8 \%)$ & \\
\hline $\begin{array}{l}\text { AJCC staging } \\
\text { (overall) }\end{array}$ & & & & & & & 0.096 \\
\hline p-Stage III & 82 & $(24.6 \%)$ & 41 & $(29.3 \%)$ & 41 & $(21.2 \%)$ & \\
\hline p-Stage IV & 251 & $(75.4 \%)$ & 99 & $(70.7 \%)$ & 152 & $(78.8 \%)$ & \\
\hline Extracapsular spread & & & & & & & 0.370 \\
\hline No & 140 & $(42.0 \%)$ & 63 & $(45.0 \%)$ & 77 & $(39.9 \%)$ & \\
\hline Yes & 193 & $(58.0 \%)$ & 77 & $(55.0 \%)$ & 116 & $(60.1 \%)$ & \\
\hline Differentiation & & & & & & & 0.929 \\
\hline Well & 60 & $(18.0 \%)$ & 24 & $(17.1 \%)$ & 36 & $(18.7 \%)$ & \\
\hline Moderate & 220 & $(66.1 \%)$ & 93 & $(66.4 \%)$ & 127 & $(65.8 \%)$ & \\
\hline Poor & 53 & $(15.9 \%)$ & 23 & $(16.4 \%)$ & 30 & $(15.5 \%)$ & \\
\hline $\begin{array}{l}\text { Bone marrow } \\
\text { invasion }\end{array}$ & & & & & & & 0.218 \\
\hline No & 264 & $(79.3 \%)$ & 116 & $(82.9 \%)$ & 148 & $(76.7 \%)$ & \\
\hline Yes & 69 & $(20.7 \%)$ & 24 & $(17.1 \%)$ & 45 & $(23.3 \%)$ & \\
\hline Skin invasion & & & & & & & 0.716 \\
\hline No & 299 & $(89.8 \%)$ & 127 & $(90.7 \%)$ & 172 & $(89.1 \%)$ & \\
\hline Yes & 34 & $(10.2 \%)$ & 13 & $(9.3 \%)$ & 21 & $(10.9 \%)$ & \\
\hline Perineural invasion & & & & & & & 1.000 \\
\hline No & 162 & $(48.6 \%)$ & 68 & $(48.6 \%)$ & 94 & $(48.7 \%)$ & \\
\hline Yes & 171 & $(51.4 \%)$ & 72 & $(51.4 \%)$ & 99 & $(51.3 \%)$ & \\
\hline Vascular invasion & & & & & & & 0.812 \\
\hline No & 315 & $(94.6 \%)$ & 132 & $(94.3 \%)$ & 183 & $(94.8 \%)$ & \\
\hline Yes & 18 & $(5.4 \%)$ & 8 & $(5.7 \%)$ & 10 & $(5.2 \%)$ & \\
\hline Lymphatic invasion & & & & & & & 0.619 \\
\hline No & 291 & $(87.4 \%)$ & 124 & $(88.6 \%)$ & 167 & $(86.5 \%)$ & \\
\hline Yes & 42 & $(12.6 \%)$ & 16 & $(11.4 \%)$ & 26 & $(13.5 \%)$ & \\
\hline Margin status & & & & & & & 0.017 \\
\hline$<5 \mathrm{~mm}$ & 41 & $(12.3 \%)$ & 10 & $(7.1 \%)$ & 31 & $(16.1 \%)$ & \\
\hline$\geq 5 \mathrm{~mm}$ & 288 & $(86.5 \%)$ & 129 & $(92.1 \%)$ & 159 & $(82.4 \%)$ & \\
\hline Unknown & 4 & $(1.2 \%)$ & 1 & $(0.7 \%)$ & 3 & $(1.6 \%)$ & \\
\hline
\end{tabular}

(Continued) 


\begin{tabular}{|c|c|c|c|c|c|c|c|}
\hline \multirow[t]{2}{*}{ Characteristics } & \multicolumn{2}{|c|}{ Entire cohort } & \multicolumn{2}{|c|}{ TP53 Wt } & \multicolumn{2}{|c|}{ TP53 mutations } & \multirow[t]{2}{*}{ P value } \\
\hline & $\mathbf{N}$ & $(\%)$ & $\mathbf{N}$ & $(\%)$ & $\mathbf{N}$ & $(\%)$ & \\
\hline Tumor depth & & & & & & & 0.127 \\
\hline$<10 \mathrm{~mm}$ & 111 & $(33.3 \%)$ & 53 & $(37.9 \%)$ & 58 & $(30.1 \%)$ & \\
\hline$\geq 10 \mathrm{~mm}$ & 221 & $(66.4 \%)$ & 86 & $(61.4 \%)$ & 135 & $(69.9 \%)$ & \\
\hline Unknown & 1 & $(0.3 \%)$ & 1 & $(0.7 \%)$ & 0 & $(0.0 \%)$ & \\
\hline Local recurrence & & & & & & & 0.781 \\
\hline No & 267 & $(80.2 \%)$ & 111 & $(79.3 \%)$ & 156 & $(80.8 \%)$ & \\
\hline Yes & 66 & $(19.8 \%)$ & 29 & $(20.7 \%)$ & 37 & $(19.2 \%)$ & \\
\hline Neck recurrence & & & & & & & 0.795 \\
\hline No & 254 & $(76.3 \%)$ & 108 & $(77.1 \%)$ & 146 & $(75.6 \%)$ & \\
\hline Yes & 79 & $(23.7 \%)$ & 32 & $(22.9 \%)$ & 47 & $(24.4 \%)$ & \\
\hline Distant metastasis & & & & & & & 0.002 \\
\hline No & 252 & $(75.7 \%)$ & 118 & $(84.3 \%)$ & 134 & $(69.4 \%)$ & \\
\hline Yes & 81 & $(24.3 \%)$ & 22 & $(15.7 \%)$ & 59 & $(30.6 \%)$ & \\
\hline $\begin{array}{l}\text { Level IV/V } \\
\text { metastases }\end{array}$ & & & & & & & 0.400 \\
\hline No & 308 & $(92.5 \%)$ & 132 & $(94.3 \%)$ & 176 & $(91.2 \%)$ & \\
\hline Yes & 25 & $(7.5 \%)$ & 8 & $(5.7 \%)$ & 17 & $(8.8 \%)$ & \\
\hline $\begin{array}{l}\text { Second primary } \\
\text { tumor }\end{array}$ & & & & & & & 0.257 \\
\hline No & 270 & $(81.1 \%)$ & 118 & $(84.3 \%)$ & 152 & $(78.8 \%)$ & \\
\hline Yes & 63 & $(18.9 \%)$ & 22 & $(15.7 \%)$ & 41 & $(21.2 \%)$ & \\
\hline $\begin{array}{l}\text { Relapse after } \\
\text { complete treatment }\end{array}$ & & & & & & & 0.121 \\
\hline No & 171 & $(51.4 \%)$ & 79 & $(56.4 \%)$ & 92 & $(47.7 \%)$ & \\
\hline Yes & 162 & $(48.6 \%)$ & 61 & $(43.6 \%)$ & 101 & $(52.3 \%)$ & \\
\hline OSCC-related death & & & & & & & 0.007 \\
\hline No & 197 & $(59.2 \%)$ & 95 & $(67.9 \%)$ & 102 & $(52.8 \%)$ & \\
\hline Yes & 136 & $(40.8 \%)$ & 45 & $(32.1 \%)$ & 91 & $(47.2 \%)$ & \\
\hline Death from any cause & & & & & & & 0.002 \\
\hline No & 135 & $(40.5 \%)$ & 71 & $(50.7 \%)$ & 64 & $(33.2 \%)$ & \\
\hline Yes & 198 & $(59.5 \%)$ & 69 & $(49.3 \%)$ & 129 & $(66.8 \%)$ & \\
\hline
\end{tabular}

Categorical data were compared with the Fisher's exact test or the $\chi^{2}$ test, as appropriate.

results obtained for DSS, the DFS of patients bearing all other mutations was comparable to that of patients with wild-type TP53 and better than that of patients with TP53 DBD missense mutations. The difference between TP53 DBD missense mutations and all other mutations was of borderline statistical significance for both DFS and DSS (DFS: HR, 1.57; 95\% CI, 0.93-2.41; $\mathrm{P}=0.099$, DSS HR, $1.75 ; 95 \%$ CI, $0.98-2.68 ; \mathrm{P}=0.065)$. Similar to DFS and DSS, OS was lower for patients carrying TP53 DBD missense mutations compared with wild-type TP53 (HR, 1.64; 95\% CI, 1.23-2.22; $\mathrm{P}=0.001$ ). In patients with all other mutations, five cases of death occurring after a 
follow-up of more than 100 months led to a decreased OS. Because these deaths were unrelated to the primary tumor, we reasoned that second primary tumors might have been a contributing factor. The percentage of second primary tumors was nonsignificantly higher in patients with all other mutations compared with wild-type TP53 (27.8\% and $15.7 \%$, respectively, $\mathrm{P}=0.143$ ). A second primary tumor was observed in three of the five patients with all
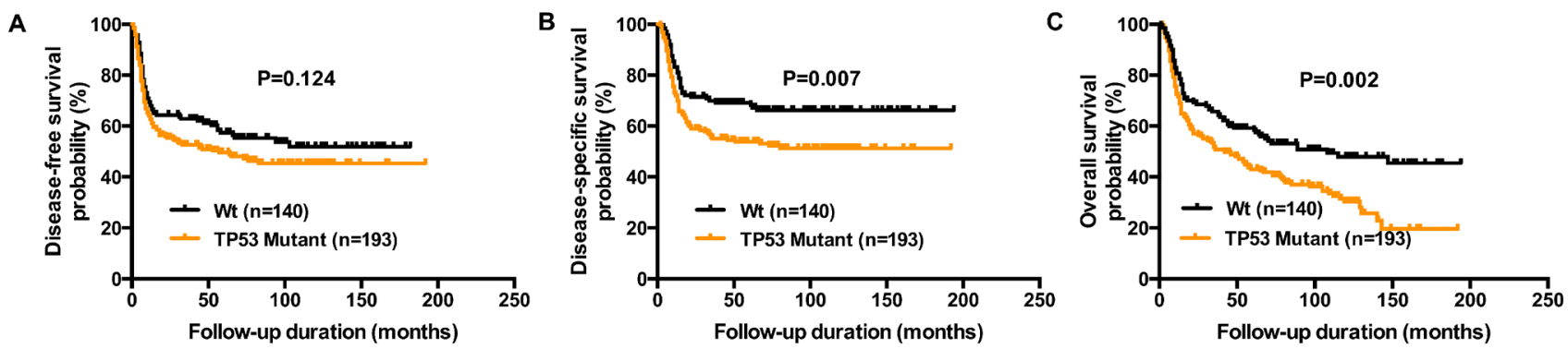

Figure 2: Reduced DFS, DSS, and OS in OSCC patients harboring TP53 mutations. Kaplan-Meier plots depict DFS panel A., DSS panel B., and OS panel C. of 333 OSCC patients according to the presence of any TP53 mutation versus wild-type TP53. P values were calculated with the log-rank test.

A
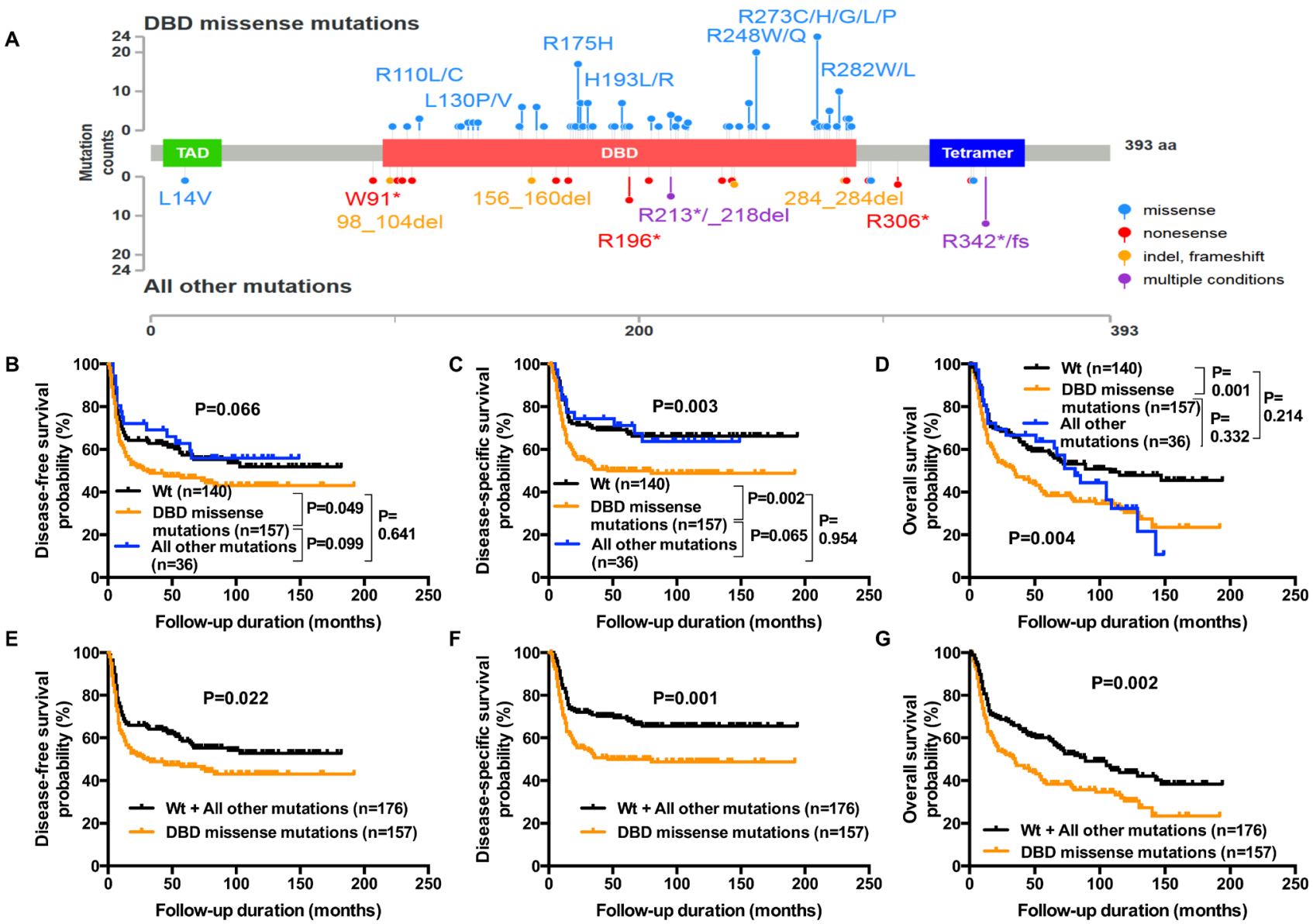

Figure 3: Reduced DFS, DSS, and OS in OSCC patients harboring TP53 DBD missense mutations. Missense TP53 mutations in the DNA binding domain (residues 95-289) were considered as "TP53 DBD missense mutations", whereas all of the remaining mutations were defined as "all other mutations". The distribution of TP53 DBD missense mutations and all other mutations in the study patients ( $\mathrm{n}=333)$ is shown in panel A. Kaplan-Meier plots depict DFS panel B., DSS panel C., and OS panel D. of patients with TP53 DBD missense mutations versus all other mutations. Patients with all other mutations and wild-type TP53 were then merged into a single group and compared with patients carrying TP53 DBD missense mutations. After grouping, Kaplan-Meier plots of DFS panel E., DSS panel F., and OS panel G. were constructed. P values were calculated with the log-rank test. 
Table 2: TP53 mutations and DSS according to different TP53 mutation classifications

\begin{tabular}{|c|c|c|c|c|c|}
\hline TP53 mutation status & $\mathbf{N}$ & $(\%)$ & HR & $95 \% \mathrm{CI}$ & P Value \\
\hline Wild-type & 140 & $42.0 \%$ & 1.00 & & \\
\hline DBD missense mutations & 157 & $47.1 \%$ & 1.78 & $1.23-2.57$ & 0.002 \\
\hline All other mutations & 36 & $10.8 \%$ & 1.02 & $0.54-1.93$ & 0.955 \\
\hline Wild-type & 140 & $42.0 \%$ & 1.00 & & \\
\hline Evolutionary action score high & 111 & $33.3 \%$ & 1.99 & $1.35-2.93$ & 0.001 \\
\hline Evolutionary action score low & 49 & $14.7 \%$ & 1.49 & $0.90-2.49$ & 0.124 \\
\hline Wild-type & 140 & $42.0 \%$ & 1 & & \\
\hline L2, L3, LSH & 133 & $39.9 \%$ & 1.78 & $1.21-2.59$ & 0.003 \\
\hline Mutation outside of L2,L3,LSH & 60 & $18.0 \%$ & 1.31 & $0.80-2.16$ & 0.276 \\
\hline Wild-type & 140 & $42.0 \%$ & 1.00 & & \\
\hline Disruptive & 81 & $24.3 \%$ & 1.38 & $0.88-2.15$ & 0.158 \\
\hline Nondisruptive & 112 & $33.6 \%$ & 1.82 & $1.23-2.69$ & 0.003 \\
\hline Wild-type & 140 & $42.0 \%$ & & & \\
\hline Truncating & 44 & $13.2 \%$ & 1.45 & $0.85-2.48$ & 0.177 \\
\hline Non-truncating & 149 & $44.7 \%$ & 1.68 & $1.16-2.43$ & 0.007 \\
\hline
\end{tabular}

$\mathrm{P}$ values were calculated with univariate Cox regression analysis.

other mutations who died after $>100$ months of follow-up. Despite the decreased OS observed during the late followup period, the OS of patients with all other mutations and wild-type TP53 did not differ significantly from each other $(\mathrm{P}=0.214)$.

Because of their similar survival characteristics (especially in terms of DFS and DSS), patients with all other mutations and wild-type TP53 were grouped together for the purpose of analysis. Compared with the combined group, patients with TP53 DBD missense mutations were found to have significantly shorter DFS, DSS, and OS (DFS: HR, 1.42; 95\% CI, 1.06-1.97; $\mathrm{P}=0.022$, DSS: HR, 1.78; 95\% CI, 1.29-2.54; $\mathrm{P}=0.001$; OS: HR, 1.55; 95\% CI, 1.19-2.10; $\mathrm{P}=0.002$; Figure $3 \mathrm{E}-3 \mathrm{G})$. The distribution of the majority of risk factors was similar in patients with all other mutations and wild-type TP53, confirming that the two groups were biologically comparable. Compared with patients with TP53 DBD missense mutations, those with all other mutations and wild-type TP53 had a lower AJCC T-classification $(\mathrm{P}=0.036)$, AJCC overall stage $(\mathrm{P}=0.016)$, a lower risk of bone marrow invasion $(\mathrm{P}=0.014)$, and distant metastases $(\mathrm{P}<0.001$; Table 3$)$.

\section{TP53 DBD missense mutations are an independent prognostic factor for reduced DSS}

We next sought to identify the prognostic factors for DSS (Table 4). In univariate analysis, we identified advanced AJCC T-classification, N-classification, and overall stage, ECS, and TP53 DBD missense mutations as the main risk factors for DSS ( $\mathrm{P} \leq 0.001)$. Other factors included tumor differentiation, invasion to bone marrow, skin, and lymphatic vessels, as well as margin status, tumor depth, and the occurrence of any TP53 mutation. After allowance for potential confounders, multivariate analysis revealed that TP53 DBD missense mutations retained their independent prognostic significance for DSS (HR, 1.55; 95\% CI, 1.09-2.20; $\mathrm{P}=0.014)$. Other independent predictors were advanced AJCC T-classification (HR, 1.94; 95\% CI, 1.33-2.81; $\mathrm{P}=0.001$ ), $\mathrm{N}$-classification (HR, 1.55; 95\% CI, 1.01-2.38; $\mathrm{P}=0.047$ ), and the presence of ECS (HR, 1.69; 95\% CI, 1.12-2.55; $\mathrm{P}=0.013$ ). 
Table 3: General characteristics of patients according to the type of TP53 mutations

\begin{tabular}{|c|c|c|c|c|c|c|c|c|c|}
\hline \multirow[t]{2}{*}{ Characteristics } & \multicolumn{2}{|c|}{ TP53 Wt } & \multicolumn{2}{|c|}{$\begin{array}{l}\text { TP53 All other } \\
\text { mutations }\end{array}$} & \multicolumn{2}{|c|}{$\begin{array}{c}\text { TP53 Wt/All } \\
\text { other mutations }\end{array}$} & \multicolumn{2}{|c|}{$\begin{array}{c}\text { TP53 DBD missense } \\
\text { mutations }\end{array}$} & \multirow[t]{2}{*}{ P value } \\
\hline & $\mathbf{N}$ & $(\%)$ & $\mathbf{N}$ & $(\%)$ & $\mathbf{N}$ & $(\%)$ & $\mathbf{N}$ & $(\%)$ & \\
\hline Entire study cohort & 140 & $(42.0 \%)$ & 36 & $(10.8 \%)$ & 176 & $(52.8 \%)$ & 157 & $(47.1 \%)$ & \\
\hline Sex & & & & & & & & & 1.000 \\
\hline Male & 129 & $(92.1 \%)$ & 36 & $(100.0 \%)$ & 165 & $(93.8 \%)$ & 148 & $(94.3 \%)$ & \\
\hline Female & 11 & $(7.9 \%)$ & 0 & $(0.0 \%)$ & 11 & $(6.3 \%)$ & 9 & $(5.7 \%)$ & \\
\hline \multicolumn{10}{|l|}{ Age, years } \\
\hline Median & 47 & & 47.5 & & 47 & & 49 & & \\
\hline Range & 29 & -89 & 31 & -71 & 29 & -89 & 27 & -83 & \\
\hline Mean \pm SD & 49.5 & \pm 11.7 & 48.9 & \pm 11.1 & 49.4 & \pm 11.5 & 50.1 & \pm 10.5 & \\
\hline Age (categorical) & & & & & & & & & 0.389 \\
\hline$<65$ years & 120 & $(85.7 \%)$ & 33 & $(91.7 \%)$ & 153 & $(86.9 \%)$ & 142 & $(90.4 \%)$ & \\
\hline$\geq 65$ years & 20 & $(14.3 \%)$ & 3 & $(8.3 \%)$ & 23 & $(13.1 \%)$ & 15 & $(9.6 \%)$ & \\
\hline Cigarette smoking & & & & & & & & & 0.463 \\
\hline No & 15 & $(10.7 \%)$ & 4 & $(11.1 \%)$ & 19 & $(10.8 \%)$ & 13 & $(8.3 \%)$ & \\
\hline Yes & 125 & $(89.3 \%)$ & 32 & $(88.9 \%)$ & 157 & $(89.2 \%)$ & 144 & $(91.7 \%)$ & \\
\hline Betel chewing & & & & & & & & & 0.261 \\
\hline No & 30 & $(21.4 \%)$ & 7 & $(19.4 \%)$ & 37 & $(21.0 \%)$ & 25 & $(15.9 \%)$ & \\
\hline Yes & 110 & $(78.6 \%)$ & 29 & $(80.6 \%)$ & 139 & $(79.0 \%)$ & 132 & $(84.1 \%)$ & \\
\hline Alcohol drinking & & & & & & & & & 0.333 \\
\hline No & 49 & $(35.0 \%)$ & 6 & $(16.7 \%)$ & 55 & $(31.3 \%)$ & 41 & $(26.1 \%)$ & \\
\hline Yes & 91 & $(65.0 \%)$ & 30 & $(83.3 \%)$ & 121 & $(68.8 \%)$ & 116 & $(73.9 \%)$ & \\
\hline HPV16/18 positive & & & & & & & & & 0.245 \\
\hline No & 111 & $(79.3 \%)$ & 29 & $(80.6 \%)$ & 140 & $(79.5 \%)$ & 135 & $(86.0 \%)$ & \\
\hline Yes & 20 & $(14.3 \%)$ & 6 & $(16.7 \%)$ & 26 & $(14.8 \%)$ & 16 & $(10.2 \%)$ & \\
\hline Unknown & 9 & $(6.4 \%)$ & 1 & $(2.8 \%)$ & 10 & $(5.7 \%)$ & 6 & $(3.8 \%)$ & \\
\hline Tumor site & & & & & & & & & 0.175 \\
\hline Buccal & 61 & $(43.6 \%)$ & 16 & $(44.4 \%)$ & 77 & $(43.8 \%)$ & 50 & $(31.8 \%)$ & \\
\hline Retromolar Trigone & 3 & $(2.1 \%)$ & 1 & $(2.8 \%)$ & 4 & $(2.3 \%)$ & 11 & $(7.0 \%)$ & \\
\hline Lip & 1 & $(0.7 \%)$ & 0 & $(0.0 \%)$ & 1 & $(0.6 \%)$ & 1 & $(0.6 \%)$ & \\
\hline Tongue & 46 & $(32.9 \%)$ & 15 & $(41.7 \%)$ & 61 & $(34.7 \%)$ & 64 & $(40.8 \%)$ & \\
\hline Alveolar ridge & 21 & $(15.0 \%)$ & 2 & $(5.6 \%)$ & 23 & $(13.1 \%)$ & 20 & $(12.7 \%)$ & \\
\hline Hard palate & 2 & $(1.4 \%)$ & 0 & $(0.0 \%)$ & 2 & $(1.1 \%)$ & 4 & $(2.5 \%)$ & \\
\hline Mouth floor & 6 & $(4.3 \%)$ & 2 & $(5.6 \%)$ & 8 & $(4.5 \%)$ & 7 & $(4.5 \%)$ & \\
\hline AJCC T-classification & & & & & & & & & 0.036 \\
\hline pT1-2 & 69 & $(49.3 \%)$ & 19 & $(52.8 \%)$ & 88 & $(50.0 \%)$ & 60 & $(38.2 \%)$ & \\
\hline pT3-4 & 71 & $(50.7 \%)$ & 17 & $(47.2 \%)$ & 88 & $(50.0 \%)$ & 97 & $(61.8 \%)$ & \\
\hline
\end{tabular}




\begin{tabular}{|c|c|c|c|c|c|c|c|c|c|}
\hline \multirow[t]{2}{*}{ Characteristics } & \multicolumn{2}{|c|}{ TP53 Wt } & \multicolumn{2}{|c|}{$\begin{array}{l}\text { TP53 All other } \\
\text { mutations }\end{array}$} & \multicolumn{2}{|c|}{$\begin{array}{c}\text { TP53 Wt/All } \\
\text { other mutations }\end{array}$} & \multicolumn{2}{|c|}{$\begin{array}{c}\text { TP53 DBD missense } \\
\text { mutations }\end{array}$} & \multirow[t]{2}{*}{ P value } \\
\hline & $\mathbf{N}$ & $(\%)$ & $\mathbf{N}$ & $(\%)$ & $\mathbf{N}$ & $(\%)$ & $\mathbf{N}$ & $(\%)$ & \\
\hline AJCC N-classification & & & & & & & & & 0.068 \\
\hline pN1 & 55 & $(39.3 \%)$ & 16 & $(44.4 \%)$ & 71 & $(40.3 \%)$ & 48 & $(30.6 \%)$ & \\
\hline $\mathrm{pN} 2$ & 85 & $(60.7 \%)$ & 20 & $(55.6 \%)$ & 105 & $(59.7 \%)$ & 109 & $(69.4 \%)$ & \\
\hline $\begin{array}{l}\text { AJCC staging } \\
\text { (overall) }\end{array}$ & & & & & & & & & 0.016 \\
\hline p-Stage III & 41 & $(29.3 \%)$ & 12 & $(33.3 \%)$ & 53 & $(30.1 \%)$ & 29 & $(18.5 \%)$ & \\
\hline p-Stage IV & 99 & $(70.7 \%)$ & 24 & $(66.7 \%)$ & 123 & $(69.9 \%)$ & 128 & $(81.5 \%)$ & \\
\hline Extracapsular spread & & & & & & & & & 0.122 \\
\hline No & 63 & $(45.0 \%)$ & 18 & $(50.0 \%)$ & 81 & $(46.0 \%)$ & 59 & $(37.6 \%)$ & \\
\hline Yes & 77 & $(55.0 \%)$ & 18 & $(50.0 \%)$ & 95 & $(54.0 \%)$ & 98 & $(62.4 \%)$ & \\
\hline Differentiation & & & & & & & & & 0.870 \\
\hline Well & 24 & $(17.1 \%)$ & 9 & $(25.0 \%)$ & 33 & $(18.8 \%)$ & 27 & $(17.2 \%)$ & \\
\hline Moderate & 93 & $(66.4 \%)$ & 21 & $(58.3 \%)$ & 114 & $(64.8 \%)$ & 106 & $(67.5 \%)$ & \\
\hline Poor & 23 & $(16.4 \%)$ & 6 & $(16.7 \%)$ & 29 & $(16.5 \%)$ & 24 & $(15.3 \%)$ & \\
\hline $\begin{array}{l}\text { Bone marrow } \\
\text { invasion }\end{array}$ & & & & & & & & & 0.014 \\
\hline No & 116 & $(82.9 \%)$ & 33 & $(91.7 \%)$ & 149 & $(84.7 \%)$ & 115 & $(73.2 \%)$ & \\
\hline Yes & 24 & $(17.1 \%)$ & 3 & $(8.3 \%)$ & 27 & $(15.3 \%)$ & 42 & $(26.8 \%)$ & \\
\hline Skin invasion & & & & & & & & & 0.365 \\
\hline No & 127 & $(90.7 \%)$ & 34 & $(94.4 \%)$ & 161 & $(91.5 \%)$ & 138 & $(87.9 \%)$ & \\
\hline Yes & 13 & $(9.3 \%)$ & 2 & $(5.6 \%)$ & 15 & $(8.5 \%)$ & 19 & $(12.1 \%)$ & \\
\hline Perineural invasion & & & & & & & & & 0.743 \\
\hline No & 68 & $(48.6 \%)$ & 16 & $(44.4 \%)$ & 84 & $(47.7 \%)$ & 78 & $(49.7 \%)$ & \\
\hline Yes & 72 & $(51.4 \%)$ & 20 & $(55.6 \%)$ & 92 & $(52.3 \%)$ & 79 & $(50.3 \%)$ & \\
\hline Vascular invasion & & & & & & & & & 0.629 \\
\hline No & 132 & $(94.3 \%)$ & 33 & $(91.7 \%)$ & 165 & $(93.8 \%)$ & 150 & $(95.5 \%)$ & \\
\hline Yes & 8 & $(5.7 \%)$ & 3 & $(8.3 \%)$ & 11 & $(6.3 \%)$ & 7 & $(4.5 \%)$ & \\
\hline Lymphatic invasion & & & & & & & & & 0.742 \\
\hline No & 124 & $(88.6 \%)$ & 31 & $(86.1 \%)$ & 155 & $(88.1 \%)$ & 136 & $(86.6 \%)$ & \\
\hline Yes & 16 & $(11.4 \%)$ & 5 & $(13.9 \%)$ & 21 & $(11.9 \%)$ & 21 & $(13.4 \%)$ & \\
\hline Margin status & & & & & & & & & 0.065 \\
\hline$<5 \mathrm{~mm}$ & 10 & $(7.1 \%)$ & 6 & $(16.7 \%)$ & 16 & $(9.1 \%)$ & 25 & $(15.9 \%)$ & \\
\hline$\geq 5 \mathrm{~mm}$ & 129 & $(92.1 \%)$ & 30 & $(83.3 \%)$ & 159 & $(90.3 \%)$ & 129 & $(82.2 \%)$ & \\
\hline Unknown & 1 & $(0.7 \%)$ & 0 & $(0.0 \%)$ & 1 & $(0.6 \%)$ & 3 & $(1.9 \%)$ & \\
\hline
\end{tabular}

(Continued) 


\begin{tabular}{|c|c|c|c|c|c|c|c|c|c|}
\hline \multirow[t]{2}{*}{ Characteristics } & \multicolumn{2}{|c|}{ TP53 Wt } & \multicolumn{2}{|c|}{$\begin{array}{l}\text { TP53 All other } \\
\text { mutations }\end{array}$} & \multicolumn{2}{|c|}{$\begin{array}{c}\text { TP53 Wt/All } \\
\text { other mutations }\end{array}$} & \multicolumn{2}{|c|}{$\begin{array}{l}\text { TP53 DBD missense } \\
\text { mutations }\end{array}$} & \multirow[t]{2}{*}{$P$ value } \\
\hline & $\mathbf{N}$ & $(\%)$ & $\mathbf{N}$ & $(\%)$ & $\mathbf{N}$ & $(\%)$ & $\mathbf{N}$ & $(\%)$ & \\
\hline Tumor depth & & & & & & & & & 0.244 \\
\hline$<10 \mathrm{~mm}$ & 53 & $(37.9 \%)$ & 11 & $(30.6 \%)$ & 64 & $(36.4 \%)$ & 47 & $(29.9 \%)$ & \\
\hline$\geq 10 \mathrm{~mm}$ & 86 & $(61.4 \%)$ & 25 & $(69.4 \%)$ & 111 & $(63.1 \%)$ & 110 & $(70.1 \%)$ & \\
\hline Unknown & 1 & $(0.7 \%)$ & 0 & $(0.0 \%)$ & 1 & $(0.6 \%)$ & 0 & $(0.0 \%)$ & \\
\hline Local recurrence & & & & & & & & & 1.000 \\
\hline No & 111 & $(79.3 \%)$ & 30 & $(83.3 \%)$ & 141 & $(80.1 \%)$ & 126 & $(80.3 \%)$ & \\
\hline Yes & 29 & $(20.7 \%)$ & 6 & $(16.7 \%)$ & 35 & $(19.9 \%)$ & 31 & $(19.7 \%)$ & \\
\hline Neck recurrence & & & & & & & & & 0.699 \\
\hline No & 108 & $(77.1 \%)$ & 28 & $(77.8 \%)$ & 136 & $(77.3 \%)$ & 118 & $(75.2 \%)$ & \\
\hline Yes & 32 & $(22.9 \%)$ & 8 & $(22.2 \%)$ & 40 & $(22.7 \%)$ & 39 & $(24.8 \%)$ & \\
\hline Distant metastasis & & & & & & & & & $<0.001$ \\
\hline No & 118 & $(84.3 \%)$ & 30 & $(83.3 \%)$ & 148 & $(84.1 \%)$ & 104 & $(66.2 \%)$ & \\
\hline Yes & 22 & $(15.7 \%)$ & 6 & $(16.7 \%)$ & 28 & $(15.9 \%)$ & 53 & $(33.8 \%)$ & \\
\hline $\begin{array}{l}\text { Level IV/V } \\
\text { metastases }\end{array}$ & & & & & & & & & 0.214 \\
\hline No & 132 & $(94.3 \%)$ & 34 & $(94.4 \%)$ & 166 & $(94.3 \%)$ & 142 & $(90.4 \%)$ & \\
\hline Yes & 8 & $(5.7 \%)$ & 2 & $(5.6 \%)$ & 10 & $(5.7 \%)$ & 15 & $(9.6 \%)$ & \\
\hline $\begin{array}{l}\text { Second primary } \\
\text { tumor }\end{array}$ & & & & & & & & & 0.780 \\
\hline No & 118 & $(84.3 \%)$ & 26 & $(72.2 \%)$ & 144 & $(81.8 \%)$ & 126 & $(80.3 \%)$ & \\
\hline Yes & 22 & $(15.7 \%)$ & 10 & $(27.8 \%)$ & 32 & $(18.2 \%)$ & 31 & $(19.7 \%)$ & \\
\hline $\begin{array}{l}\text { Relapse after } \\
\text { complete treatment }\end{array}$ & & & & & & & & & 0.037 \\
\hline No & 79 & $(56.4 \%)$ & 21 & $(58.3 \%)$ & 100 & $(56.8 \%)$ & 71 & $(45.2 \%)$ & \\
\hline Yes & 61 & $(43.6 \%)$ & 15 & $(41.7 \%)$ & 76 & $(43.2 \%)$ & 86 & $(54.8 \%)$ & \\
\hline OSCC-related death & & & & & & & & & 0.001 \\
\hline No & 95 & $(67.9 \%)$ & 24 & $(66.7 \%)$ & 119 & $(67.6 \%)$ & 78 & $(49.7 \%)$ & \\
\hline Yes & 45 & $(32.1 \%)$ & 12 & $(33.3 \%)$ & 57 & $(32.4 \%)$ & 79 & $(50.3 \%)$ & \\
\hline Death from any cause & & & & & & & & & 0.010 \\
\hline No & 71 & $(50.7 \%)$ & 12 & $(33.3)$ & 83 & $(47.2 \%)$ & 52 & $(33.1 \%)$ & \\
\hline Yes & 69 & $(49.3 \%)$ & 24 & $(66.7)$ & 93 & $(52.8 \%)$ & 105 & $(66.9 \%)$ & \\
\hline
\end{tabular}

Characteristics of patients with wild-type TP53, all other TP53 mutations, all other TP53 mutations combined with wildtype TP53, and TP53 DBD missense mutations. Categorical data (patients with wild-type TP53 combined with all other mutations versus patients with DBD missense mutations) were compared with the Fisher's exact test or the $\chi^{2}$ test, as appropriate. 
Table 4: Univariate and multivariate analyses of risk factors in relation to disease-specific survival

\begin{tabular}{|c|c|c|c|c|c|c|c|c|}
\hline Variable & $\mathbf{N}$ & $(\%)$ & $\mathbf{H R}^{(\mathbf{a})}$ & $95 \% \mathrm{CI}^{(\mathrm{a})}$ & P Value ${ }^{(a)}$ & $\mathbf{H R}^{(\mathbf{b})}$ & $95 \% \mathrm{CI}^{(\mathrm{b})}$ & P Value ${ }^{(b)}$ \\
\hline \multicolumn{9}{|l|}{ Risk factor } \\
\hline Sex (Male vs Female) & 313 & $(94.0 \%)$ & 1.73 & $0.71-4.22$ & 0.230 & & & \\
\hline $\begin{array}{l}\text { Age }(\geq 65 \text { years vs }<65 \\
\text { years })\end{array}$ & 38 & $(11.4 \%)$ & 0.88 & $0.51-1.53$ & 0.649 & & & \\
\hline $\begin{array}{l}\text { HPV status }(16 / 18 \\
\text { positive vs HPV } 16 / 18 \\
\text { negative) }\end{array}$ & 42 & $(12.6 \%)$ & 1.05 & $0.64-1.70$ & 0.860 & & & \\
\hline $\begin{array}{l}\text { AJCC T-classification } \\
\text { (pT3-4 vs pT1-2) }\end{array}$ & 185 & $(55.6 \%)$ & 2.31 & $1.60-3.33$ & $<0.001$ & 1.94 & $1.33-2.81$ & 0.001 \\
\hline $\begin{array}{l}\text { AJCC } \\
\text { N-classification (pN2 } \\
\text { vs pN1) }\end{array}$ & 214 & $(64.3 \%)$ & 2.22 & $1.50-3.29$ & $<0.001$ & 1.55 & $1.01-2.38$ & 0.047 \\
\hline $\begin{array}{l}\text { AJCC staging (IV vs } \\
\text { III) }\end{array}$ & 251 & $(75.4 \%)$ & 2.61 & $1.61-4.25$ & $<0.001$ & & & \\
\hline $\begin{array}{l}\text { Extracapsular spread } \\
\text { (Yes vs No) }\end{array}$ & 193 & $(58.0 \%)$ & 2.37 & $1.63-3.44$ & $<0.001$ & 1.69 & $1.12-2.55$ & 0.013 \\
\hline $\begin{array}{l}\text { Differentiation (Poor } \\
\text { vs Well/Moderate) }\end{array}$ & 53 & $(15.9 \%)$ & 1.65 & $1.09-2.51$ & 0.018 & & & \\
\hline $\begin{array}{l}\text { Bone marrow } \\
\text { invasion (Yes vs No) }\end{array}$ & 69 & $(20.7 \%)$ & 1.50 & $1.02-2.20$ & 0.040 & & & \\
\hline $\begin{array}{l}\text { Skin invasion (Yes vs } \\
\text { No) }\end{array}$ & 34 & $(10.2 \%)$ & 1.69 & $1.05-2.72$ & 0.030 & & & \\
\hline $\begin{array}{l}\text { Perineural invasion } \\
\text { (Yes vs No) }\end{array}$ & 171 & $(51.4 \%)$ & 1.27 & $0.91-1.79$ & 0.160 & & & \\
\hline $\begin{array}{l}\text { Vascular invasion } \\
\text { (Yes vs No) }\end{array}$ & 18 & $(5.4 \%)$ & 0.95 & $0.44-2.03$ & 0.892 & & & \\
\hline $\begin{array}{l}\text { Lymphatic invasion } \\
\text { (Yes vs No) }\end{array}$ & 42 & $(12.6 \%)$ & 1.85 & $1.20-2.85$ & 0.006 & & & \\
\hline $\begin{array}{l}\text { Margin status }(<5 \\
\mathrm{mm} \text { vs } \geq 5 \mathrm{~mm})\end{array}$ & 41 & $(12.3 \%)$ & 1.91 & $1.22-2.99$ & 0.005 & & & \\
\hline $\begin{array}{l}\text { Tumor depth }(\geq 10 \\
\text { mm vs }<10 \mathrm{~mm})\end{array}$ & 221 & $(66.4 \%)$ & 1.76 & $1.20-2.60$ & 0.004 & & & \\
\hline \multicolumn{9}{|l|}{ TP53 Mutation } \\
\hline Mutant vs Wildtype & 193 & $(58.0 \%)$ & 1.62 & $1.14-2.32$ & 0.008 & & & \\
\hline $\begin{array}{l}\text { DBD missense } \\
\text { mutations vs Wt/All } \\
\text { other mutations }\end{array}$ & 157 & $(47.1 \%)$ & 1.78 & $1.26-2.50$ & 0.001 & 1.55 & $1.09-2.20$ & 0.014 \\
\hline
\end{tabular}

$\mathrm{P}$ values were calculated with Cox regression using a forward selection procedure for multivariate analysis.

anivariate analysis

${ }^{\mathrm{b}}$ Multivariate analysis 


\section{TP53 DBD missense mutations combined with traditional clinical risk factors identify high-risk OSCC patients}

We finally reasoned that the difference in DSS between patients bearing TP53 DBD missense mutations and patients with all other mutations or wild-type TP53 was less than $20 \%$ (49.7\% versus $67.6 \%$, respectively). Furthermore, the high prevalence of TP53 DBD missense mutations (47.1\%) prevented a clear identification of highrisk patients. In an effort to improve patient stratification, we devised a prognostic scoring system based on the four independent predictors of DSS identified on multivariate analysis. One point was attributed to each risk factor present. Three risk categories were identified, as follows: low-risk (scores of $0-1 ; n=95,28.5 \%$ ), intermediate-risk (scores of $2-3 ; \mathrm{n}=184,55.3 \%$ ) and high-risk (score of 4; $\mathrm{n}=54,16.2 \%$ ) (Table 5 and Figure 4).

DFS, DSS, and OS of intermediate-risk and highrisk patients were significantly lower than those observed in low-risk patients (all $\mathrm{P}<0.001$ ). Specifically, the hazard ratios for DFS, DSS, and OS were 2.64, 3.22, and 1.90 for intermediate-risk patients, and 4.60, 6.78 and, 3.92 for high-risk patients, respectively. The DFS rates of low-, intermediate-, and high-risk patients were $73.7 \%, 46.2 \%$, and $29.6 \%$, respectively; DSS rates were $82.1 \%, 55.4 \%$, and $31.5 \%$, and OS rates were $56.8 \%, 38.0 \%$, and $20.4 \%$, respectively.

\section{DISCUSSION}

Interest into the prognostic significance of traditional $[3,20]$ and genetic $[19,21]$ risk factors in OSCC is mounting. Previous studies have shown that TP53 expression [22] and mutation status [6,7] can predict prognosis in OSCC patients. In the present study, we demonstrate that TP53 DBD missense mutations were the optimal classifier for distinguishing between TP53 mutant patients with low and high clinical risk. We also show that TP53 DBD missense mutations can be used in combination with traditional risk factors for improving prognostic stratification.

With regard to TP53 mutation subtypes, we were unable to confirm a particularly deleterious effect of truncating mutations [9]. Truncating mutations are included in the group of disruptive mutations [8], which may likely have contributed to the unsatisfactory results obtained with this classification method in our cohort. In contrast, mutations in L2, L3 or LSH $[7,16,23]$ and high-risk missense mutations according to the EAp53 $[14,15]$ were capable of identifying high-risk patients in a successful manner. We believe that this capacity may be attributed to the fact that they include a high proportion of a gain-of-function mutations [10]. However, these classification approaches were inferior to TP53 DBD missense mutations for distinguishing between high- and low-risk TP53 mutation carriers. It is feasible that this could be attributed to their lack of inclusion of all missense mutations occurring in the DBD that mediate an enhanced risk. It should be noted that EAp53 also includes missense mutations located outside of DBD. However, the vast majority of missense mutations are located within the DBD, indicating a selection for missense mutations in this region. The low number of missense mutations located outside of the DBD complicates the assessment of their prognostic impact. However, amino acid substitutions occurring outside of the DBD may have biological effects. For example, an arginine variant located at codon 72 is associated with a higher likelihood of apoptosis [24] and a later disease onset in its carriers [25].

The results of our study indicate that TP53 DBD missense mutations - but not all of the remaining mutations (defined as "all other mutations" in this study) - are significantly associated with reduced DFS, DSS, and OS in patients with advanced OSCC. However, the survival difference between patients with TP53 DBD missense mutations and all other mutations did not reach statistical significance $(\mathrm{P}=0.099$ and $\mathrm{P}=0.065$ for DFS and DSS, respectively), most likely because of the small sample size $(n=36)$ of patients harboring all other mutations. We also observed a decrease in OS for patients with all other mutations during the late followup period. Although this phenomenon was unrelated to primary disease, there was a tendency toward an increased incidence of second primary tumors in patients with all other mutations compared with wild-type TP53 ( $\mathrm{P}=0.143$ ). Future studies are required to investigate whether the observed late reduction in OS is a hallmark of patients with all other mutations and to indicate whether these patients may need a closer follow-up schedule.

It is noteworthy that nearly $50 \%$ of all patients included in the current study were carriers of TP53 DBD missense mutations. In addition, the difference in terms of DSS between these patients and patients with wildtype TP53 or all other mutations was less than $20 \%$. This hampered the identification of a specific subgroup of patients at high clinical risk. The analysis of TP53 mutations in combination with other risk factors (e.g., nodal status or $3 p$ loss) was shown to be clinically useful for predicting treatment outcomes and survival [26, 27]. We therefore devised a prognostic scoring system that combined the presence of TP53 DBD missense mutations with traditional prognostic factors. The combination of TP53 DBD missense mutations with the three independent prognostic factors for DSS identified in multivariate analysis (AJCC N-classification, AJCC T-classification, and ECS) allowed the identification of three distinct risk groups, i.e., low-risk ( $28.5 \%$ of the study patients), intermediate-risk (55.3\%), and high-risk (16.2\%) patients. We believe that such stratification can rationalize both OSCC treatment and clinical follow-up. 
Table 5: Prognostic scoring system for OSCC patient survival

\begin{tabular}{|c|c|c|c|c|c|c|c|c|c|c|c|c|c|c|}
\hline Score & $\mathbf{N}$ & $(\%)$ & DFS & HR & $\begin{array}{c}95 \% \\
\text { CI }\end{array}$ & $\begin{array}{c}\mathbf{P} \\
\text { Value }\end{array}$ & DSS & HR & $\begin{array}{c}95 \% \\
\text { CI }\end{array}$ & $\begin{array}{c}\mathbf{P} \\
\text { Value }\end{array}$ & OS & HR & $\begin{array}{c}95 \% \\
\text { CI }\end{array}$ & $\begin{array}{c}P \\
\text { Value }\end{array}$ \\
\hline $0+1$ & 95 & $(28.5 \%)$ & $73.7 \%$ & & & & $82.1 \%$ & & & & $56.8 \%$ & & & \\
\hline $2+3$ & 184 & $(55.3 \%)$ & $46.2 \%$ & 2.64 & $\begin{array}{c}1.70- \\
4.10\end{array}$ & $\begin{array}{c}< \\
0.001\end{array}$ & $55.4 \%$ & 3.22 & $\begin{array}{l}1.91- \\
5.44\end{array}$ & $\begin{array}{c}< \\
0.001\end{array}$ & $38.0 \%$ & 1.90 & $\begin{array}{l}1.33- \\
2.72\end{array}$ & $\begin{array}{c}< \\
0.001\end{array}$ \\
\hline 4 & 54 & $(16.2 \%)$ & $29.6 \%$ & 4.60 & $\begin{array}{c}2.76- \\
7.68\end{array}$ & $\begin{array}{c}< \\
0.001\end{array}$ & $31.5 \%$ & 6.78 & $\begin{array}{l}3.79- \\
12.14\end{array}$ & $\begin{array}{c}< \\
0.001\end{array}$ & $20.4 \%$ & 3.92 & $\begin{array}{c}2.53- \\
6.06\end{array}$ & $\begin{array}{c}< \\
0.001\end{array}$ \\
\hline
\end{tabular}

The scoring system is based on the four independent risk factors identified by multivariate analysis (i.e., presence of a TP53 DBD missense mutation, ECS, advanced AJCC N- and T-classifications). P values were calculated with Cox regression using patients with scores of $0-1$ as the reference category.
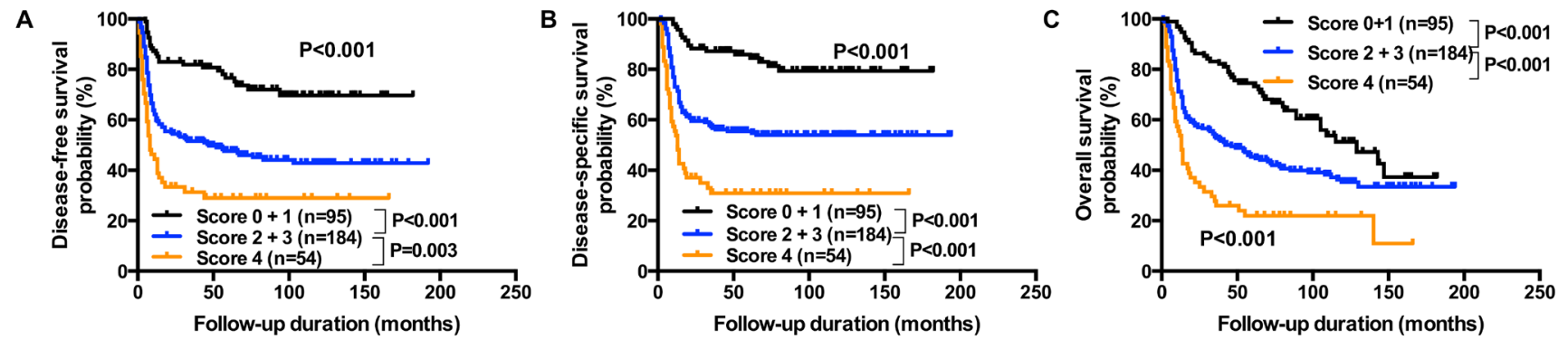

Figure 4: Prognostic scoring system for DSS. The scoring system is based on the four independent predictors of DSS identified by multivariate analysis (i.e., presence of a TP53 DBD missense mutation, ECS, advanced AJCC N- and T-classification). One point was attributed to each risk factor present. Three risk categories were identified, as follows: low-risk (scores of $0-1 ; \mathrm{n}=95,28.5 \%$ ), intermediaterisk (scores of 2-3; $n=184,55.3 \%$ ) and high-risk (score of 4; $n=54,16.2 \%$ ). Kaplan-Meier plots for DFS panel A., DSS panel B., and OS panel C. for the three risk groups were constructed. P values were calculated with the log-rank test.

Notably, TP53 mutations have been associated with a decreased response to both 5-fluorouracil [26] and radiotherapy [16]. Additionally, TP53 mutation subtypes may directly guide future targeted treatment. Viral therapy may be considered for loss-of-function mutations, whereas small molecules targeting gain-of-function mutations may be used to restore TP53 functionality [28]. Small molecules have entered human clinical trials [29], and viral therapy has shown promising results in patients with head and neck malignancies [30,31].

Some caveats of our study merit comment. First, all of the study patients were Taiwanese and the research was conducted in a betel nut chewing endemic area. In this regard, ethnicity and different combinations of environmental factors [32] - including exposure to agents other than tobacco and alcohol [33] - have been shown to influence the occurrence or the spectrum of TP53 mutations. The question as to whether our findings are generalizable to other ethnic groups deserves further scrutiny. Consequently, our scoring system should be externally validated in independent cohorts. Second, only patients with advanced carcinoma were included. It is noteworthy that TP53 DBD missense mutations were associated with a higher prevalence of AJCC stage IV disease as compared with stage III. Additional investigations on the potential prognostic effects of TP53 DBD missense mutations in patients with earlier stages of disease would be desirable. We recognize that a complete sequencing of all exons would have led to the identification of a higher number of patients with both TP53 DBD missense mutations and other mutations. In this regard, TP53 mutations at positions not covered in this study have been reported to occur in HNSCC patients [4]. Finally, our study is limited by its retrospective nature. Further research with a longitudinal design is warranted to confirm and expand our data.

In conclusion, our results demonstrate that TP53 DBD missense mutations are an independent adverse prognostic factor in patients with advanced OSCC and may improve risk stratification when combined with traditional clinicopathological parameters. Future studies are necessary to clarify whether this prognostic tool can rationalize both OSCC treatment and clinical follow-up. 


\section{MATERIALS AND METHODS}

\section{Samples}

Tumor samples were collected from 345 pathological node-positive patients with AJCC stage III or IV OSCC who were referred to the Chang Gung Memorial Hospital between 1996 and 2009. All patients were treated with radical surgery either with or without subsequent adjuvant radiotherapy/concurrent chemoradiotherapy. The study protocol complied with the tenets of the Helsinki declaration and was approved by the Institutional Review Board of the Chang Gung Memorial Hospital (CGMH 101-4457B). Because of the retrospective nature of the study, the need for patient consent was waived.

\section{Mutation analysis of TP53}

The mutation analysis of TP53 has been previously published as part of a large genomic OSCC study [19] and the samples analyzed in the current study are the same reported previously. However, the association of different TP53 mutations with survival was not specifically analyzed. Genomic DNA was extracted from FFPE samples with the QIAamp DNA FFPE Tissue Kit (Qiagen, Hilden, Germany) according to the manufacturer's instructions. The Quant-iT'TM dsDNA HS Assay (Invitrogen, Carlsbad, CA, USA) was used for quantification of isolated DNA. The generation of target amplicon libraries was performed with the Ion AmpliSeq ${ }^{\mathrm{TM}}$ cancer panel primer pool and Ion AmpliSeq kit 2.0 (Applied Biosystems, Foster City, CA, USA) according to the manufacturer's protocol. Genomic DNA (20 ng) served as a template for multiplex PCR. The TP53 amplicons covered 51\% of the 393 aminoacids (including exons 2, 4-8 and 10) as previously described [19]. PCR reactions were followed by ligation to barcode adapters and five amplification cycles. The libraries were used for emulsion PCR (emPCR) amplification using Ion Sphere ${ }^{\mathrm{TM}}$ particles on an Ion OneTouch System (Applied Biosystems). Samples were sequenced on an Ion 318 Chip (Applied Biosystems) using the Ion Personal Genome Machine (PGM) following the manufacturer's instructions. An alignment with the hg19 reference genome was performed for data analysis, followed by the identification of genetic variants. To this aim, the Ion Torrent Suite software (v. 3.2) and the Torrent Variant Caller software (v. 3.2) were used.

\section{Mutation classification}

We annotated all variants located in exons with an allelic count $\geq 25 \times$ and an allelic frequency $\geq 5 \%$. Annotation was performed with the ANNOVAR and Cancer panel analysis pipeline (CPAP). Common single nucleotide polymorphisms without known clinical relevance were identified with the dbSNP138 database and subsequently disregarded. Only non-synonymous mutations were included in further analyses. The following TP53 mutation classifiers were analyzed in relation to their prognostic impact: 1) TP53 DBD missense mutations; 2) high-risk EAp53 mutations; 3) mutations in the L2, L3 or LSH regions; 4) disruptive mutations; and 5) truncating mutations. When multiple mutations were identified in the same patient, the presence of at least one TP53 mutation deemed to be deleterious for the corresponding classifier was sufficient for considering the subject as a mutation carrier. The classifiers were defined as follows: 1) "TP53 DBD missense mutations" were defined as missense mutations the residues 95-289, whereas all of the remaining mutations were defined as "all other mutations"; 2) mutations were classified as EAp53 highrisk and low-risk according to a previously published methodology [14]; 3) mutations in the L2, L3 or LSH regions were defined as mutations of the residues 164-194 (L2), 237-250 (L3), and 119-135 or 272-287 (LSH); 4) disruptive mutations were identified as previously described [8], and 5) all frameshift, nonsense, and splicesite mutations were considered as "truncating".

\section{Statistical analysis}

Categorical data were compared with the Fisher's exact test $(2 \times 2$ contingency tables $)$ or the $\chi^{2}$ test, as appropriate. Disease-free survival (DFS), disease-specific survival (DSS), and overall survival (OS) curves were plotted with the Kaplan-Meier method and compared with the log-rank test. DFS was defined as the time between surgery and TNM stage recurrence or the date of the last follow-up. DSS was calculated as the time from surgery to the date of death related to primary OSCC or the last follow-up. OS was defined as the time between surgery and death from any cause or the last follow-up. Univariate and multivariate analyses of DSS were based on Cox regression models. The following 17 clinicopathological variables were included in the analysis: age, sex, HPV16/18 infections, pathological AJCC T-classification, N-classification, and overall stage, ECS, differentiation, invasion to bone marrow, skin, nerve, blood vessel or lymphatic vessel, pathological margin status, tumor depth, presence of any TP53 mutation, and presence of a TP53 DBD missense mutation. The forward selection method was applied for multivariate analysis. Results were expressed as hazard ratios (HRs) with 95\% confidence intervals (CIs). We then devised a prognostic scoring system based on the four independent predictors of DSS identified on multivariate analysis (i.e., presence of a TP53 DBD missense mutation, ECS, advanced AJCC $\mathrm{N}$-classification, and advanced AJCC T-classification). One point was attributed to each risk factor present. DFS and DSS curves of low-risk (scores of 0-1), intermediaterisk (scores of 2-3) and high-risk (score of 4) patients were summarized with the Kaplan-Meier method and 
compared using the log-rank test. All calculations were performed with the GraphPad Prism (v. 6.0; GraphPad Inc., San Diego, CA, USA) and SPSS (v. 20.0.0; IBM, Somers, NY, USA) statistical packages.

\section{ACKNOWLEDGMENTS}

We thank the Linkou Chang Gung Memorial Hospital Cancer Center databank and the Bioinformatics Core and Genomic Core of the Molecular Medicine Research Center at Chang Gung University for their support and contributions.

\section{CONFLICTS OF INTEREST}

Nina Lapke, Yen-Jung Lu, and Shu-Jen Chen are employees of ACT Genomics.

\section{GRANT SUPPORT}

This work was supported by grants NMRPG3B0403, CIRPG1E0012, CMRPG3C0833 and CMRPG3D1071 from the Chang Gung Memorial Hospital at Linkou.

\section{REFERENCES}

1. Blot WJ, McLaughlin JK, Winn DM, Austin DF, Greenberg RS, Preston-Martin S, Bernstein L, Schoenberg JB, Stemhagen A, Fraumeni JF, Jr. Smoking and drinking in relation to oral and pharyngeal cancer. Cancer research. 1988; 48:3282-3287.

2. Lin YS, Jen YM, Wang BB, Lee JC, Kang BH. Epidemiology of oral cavity cancer in taiwan with emphasis on the role of betel nut chewing. ORL. 2005; 67:230-236

3. Liao CT, Lee LY, Huang SF, Chen IH, Kang CJ, Lin CY, Fan KH, Wang HM, Ng SH, Yen TC. Outcome analysis of patients with oral cavity cancer and extracapsular spread in neck lymph nodes. International journal of radiation oncology, biology, physics. 2011; 81:930-937.

4. Cancer Genome Atlas N. Comprehensive genomic characterization of head and neck squamous cell carcinomas. Nature. 2015; 517:576-582.

5. India Project Team of the International Cancer Genome C. Mutational landscape of gingivo-buccal oral squamous cell carcinoma reveals new recurrently-mutated genes and molecular subgroups. Nature communications. 2013; 4:2873.

6. Tanuma J, Izumo T, Hirano M, Oyazato Y, Hori F, Umemura E, Shisa H, Hiai H, Kitano M. FGFR4 polymorphism, TP53 mutation, and their combinations are prognostic factors for oral squamous cell carcinoma. Oncology reports. 2010; 23:739-744.

7. Zanaruddin SN, Yee PS, Hor SY, Kong YH, Ghani WM, Mustafa WM, Zain RB, Prime SS, Rahman ZA, Cheong
SC. Common oncogenic mutations are infrequent in oral squamous cell carcinoma of Asian origin. PloS one. 2013; 8:e80229.

8. Poeta ML, Manola J, Goldwasser MA, Forastiere A, Benoit N, Califano JA, Ridge JA, Goodwin J, Kenady D, Saunders J, Westra W, Sidransky D, Koch WM. TP53 mutations and survival in squamous-cell carcinoma of the head and neck. The New England journal of medicine. 2007; 357:2552-2561.

9. Lindenbergh-van der Plas M, Brakenhoff RH, Kuik DJ, Buijze M, Bloemena E, Snijders PJ, Leemans CR, Braakhuis BJ. Prognostic significance of truncating TP53 mutations in head and neck squamous cell carcinoma. Clinical cancer research. 2011; 17:3733-3741.

10. Muller PA, Vousden KH. Mutant p53 in cancer: new functions and therapeutic opportunities. Cancer cell. 2014; 25:304-317.

11. Gohler T, Jager S, Warnecke G, Yasuda H, Kim E, Deppert W. Mutant p53 proteins bind DNA in a DNA structure-selective mode. Nucleic acids research. 2005; 33:1087-1100.

12. Gaiddon C, Lokshin M, Ahn J, Zhang T, Prives C. A subset of tumor-derived mutant forms of p53 downregulate p63 and p 73 through a direct interaction with the p53 core domain. Molecular and cellular biology. 2001; 21:1874-1887.

13. Vegran F, Rebucci M, Chevrier S, Cadouot M, Boidot R, Lizard-Nacol S. Only missense mutations affecting the DNA binding domain of p53 influence outcomes in patients with breast carcinoma. PloS one. 2013; 8:e55103.

14. Neskey DM, Osman AA, Ow TJ, Katsonis P, McDonald T, Hicks SC, Hsu TK, Pickering CR, Ward A, Patel A, Yordy JS, Skinner HD, Giri U, et al. Evolutionary Action Score of TP53 Identifies High-Risk Mutations Associated with Decreased Survival and Increased Distant Metastases in Head and Neck Cancer. Cancer research. 2015; 75:1527-1536.

15. Osman AA, Neskey DM, Katsonis P, Patel AA, Ward AM, Hsu TK, Hicks SC, McDonald TO, Ow TJ, Alves MO, Pickering CR, Skinner HD, Zhao M, et al. Evolutionary Action Score of TP53 Coding Variants Is Predictive of Platinum Response in Head and Neck Cancer Patients. Cancer research. 2015; 75:1205-1215.

16. Peltonen JK, Vahakangas KH, Helppi HM, Bloigu R, Paakko P, Turpeenniemi-Hujanen T. Specific TP53 mutations predict aggressive phenotype in head and neck squamous cell carcinoma: a retrospective archival study. Head \& neck oncology. 2011; 3:20.

17. Erber R, Conradt C, Homann N, Enders C, Finckh M, Dietz A, Weidauer H, Bosch FX. TP53 DNA contact mutations are selectively associated with allelic loss and have a strong clinical impact in head and neck cancer. Oncogene. 1998; 16:1671-1679.

18. Blandino G, Levine AJ, Oren M. Mutant p53 gain of function: differential effects of different p53 mutants on 
resistance of cultured cells to chemotherapy. Oncogene. 1999; 18:477-485.

19. Chen SJ, Liu H, Liao CT, Huang PJ, Huang Y, Hsu A, Tang P, Chang YS, Chen HC, Yen TC. Ultra-deep targeted sequencing of advanced oral squamous cell carcinoma identifies a mutation-based prognostic gene signature. Oncotarget. 2015; 6:18066-18080. doi: 10.18632/oncotarget.3768.

20. Kohler HF, Kowalski LP. Prognostic impact of the level of neck metastasis in oral cancer patients. Brazilian journal of otorhinolaryngology. 2012; 78:15-20.

21. Mahdey HM, Ramanathan A, Ismail SM, Abraham MT, Jamaluddin M, Zain RB. Cyclin D1 amplification in tongue and cheek squamous cell carcinoma. Asian Pacific journal of cancer prevention. 2011; 12:2199-2204.

22. Carlos de Vicente J, Junquera Gutierrez LM, Zapatero AH, Fresno Forcelledo MF, Hernandez-Vallejo G, Lopez Arranz JS. Prognostic significance of p53 expression in oral squamous cell carcinoma without neck node metastases. Head \& neck. 2004; 26:22-30.

23. Yamazaki Y, Chiba I, Hirai A, Sugiura C, Notani K, Kashiwazaki H, Tei K, Totsuka Y, Fukuda H. Specific p53 mutations predict poor prognosis in oral squamous cell carcinoma. Oral oncology. 2003; 39:163-169.

24. Dumont P, Leu JI, Della Pietra AC, 3rd, George DL, Murphy M. The codon 72 polymorphic variants of $\mathrm{p} 53$ have markedly different apoptotic potential. Nat Genet. 2003; 33:357-365

25. Shi Q, Xiao K, Wei W, Zhang BY, Chen C, Xu Y, Chen LN, Song YT, Ma X, Zhang NS, Dong XP. Associations of TP53 mutations, codon 72 polymorphism and human papillomavirus in head and neck squamous cell carcinoma patients. Oncology reports. 2013; 30:2811-2819.

26. Kandioler D, Mittlbock M, Kappel S, Puhalla H, Herbst F, Langner C, Wolf B, Tschmelitsch J, Schippinger W, Steger G, Hofbauer F, Samonigg H, Gnant M, et al. TP53 Mutational Status and Prediction of Benefit from Adjuvant 5-Fluorouracil in Stage III Colon Cancer Patients. EBioMedicine. 2015; 2:823-828.
27. Gross AM, Orosco RK, Shen JP, Egloff AM, Carter H, Hofree M, Choueiri M, Coffey CS, Lippman SM, Hayes DN, Cohen EE, Grandis JR, Nguyen QT, et al. Multitiered genomic analysis of head and neck cancer ties TP53 mutation to 3p loss. Nat Genet. 2014; 46:939-943.

28. Bykov VJ, Issaeva N, Shilov A, Hultcrantz M, Pugacheva E, Chumakov P, Bergman J, Wiman KG, Selivanova G. Restoration of the tumor suppressor function to mutant p53 by a low-molecular-weight compound. Nature medicine. 2002; 8:282-288.

29. Lehmann S, Bykov VJ, Ali D, Andren O, Cherif H, Tidefelt U, Uggla B, Yachnin J, Juliusson G, Moshfegh A, Paul C, Wiman KG, Andersson PO. Targeting p53 in vivo: a firstin-human study with p53-targeting compound APR-246 in refractory hematologic malignancies and prostate cancer. Journal of clinical oncology. 2012; 30:3633-3639.

30. Xia ZJ, Chang JH, Zhang L, Jiang WQ, Guan ZZ, Liu JW, Zhang Y, Hu XH, Wu GH, Wang HQ, Chen ZC, Chen JC, Zhou QH, et al. [Phase III randomized clinical trial of intratumoral injection of E1B gene-deleted adenovirus (H101) combined with cisplatin-based chemotherapy in treating squamous cell cancer of head and neck or esophagus]. [Article in Chinese]. Ai Zheng. 2004; 23:1666-70.

31. Pan JJ, Zhang SW, Chen CB, Xiao SW, Sun Y, Liu CQ, Su X, Li DM, Xu G, Xu B, Lu YY. Effect of recombinant adenovirus-p53 combined with radiotherapy on long-term prognosis of advanced nasopharyngeal carcinoma. Journal of clinical oncology. 2009; 27:799-804.

32. Nylander K, Dabelsteen E, Hall PA. The p53 molecule and its prognostic role in squamous cell carcinomas of the head and neck. J Oral Pathol Med. 2000; 29:413-425.

33. Peltonen JK, Helppi HM, Paakko P, Turpeenniemi-Hujanen T, Vahakangas KH. p53 in head and neck cancer: functional consequences and environmental implications of TP53 mutations. Head \& neck oncology. 2010; 2:36. 